\title{
The sum-rule relation among phenomenological transport coefficients and its consequences in the analysis of collective diffusion problems $\dagger$
}

\author{
Irina V. Belova and Graeme E. Murch
}

Diffusion in Solids Group, The University of Newcastle, New South Wales 2308, Australia

Received 10th December 2003, Accepted 10th March 2004

First published as an Advance Article on the web 5th May 2004

In this paper, we discuss the sum-rule that relates the phenomenological coefficients in the multicomponent random alloy system in the case where the isolated vacancy mechanism is operating. We present applications of this sum-rule to intrinsic diffusion in multicomponent alloys, intrinsic diffusion in mixed alkali halides and demixing of multicomponent transition metal oxides in an oxygen potential gradient and an electric field. In each case, a very substantial simplification in the analysis is made possible because of the sum-rule.

\section{Introduction}

The theory of linear non-equilibrium thermodynamics provides a fundamental and unified formalism for a description of diffusion in solids. Well known major reviews in this area have been provided by Howard and Lidiard, ${ }^{1}$ Adda and Philibert, ${ }^{2}$ Philibert ${ }^{3}$ and Allnatt and Lidiard. ${ }^{4}$ An essential feature of the formalism is the appearance of a matrix of phenomenological coefficients, the so-called $L$ matrix.

Knowledge of the $\boldsymbol{L}$ matrix is highly desirable because it is force independent. However, the experimental determination of the $\boldsymbol{L}$ matrix for the solid state is difficult indeed. Considerable effort has therefore been put into finding relations between the phenomenological coefficients and measurable quantities such as the tracer diffusivities. An example of such relations are the so-called Manning relations. ${ }^{5,6}$ There has also been considerable interest in identifying relations among the phenomenological coefficients themselves with the expectation that this might reduce the amount of transport information actually necessary to describe a given diffusion situation. The first of these relations were the 'sum-rule relations' identified by Moleko and Allnatt ${ }^{7}$ for the random alloy model with diffusion occurring via isolated vacancies. Because of this sum-rule, in the random binary $\mathrm{AB}$ alloy the number of independent phenomenological coefficients is then reduced from three to one. For the random ternary ABC alloy, the number of phenomenological coefficients is reduced from six to three, and so on. Since that time, a number of other closely relations have been identified for various other models, including the dumbbell mechanism in the random alloy, ${ }^{8}$ the vacancy pair in randomly mixed alkali halides, ${ }^{9}$ the vacancy mechanism for the 4 -frequency model for ordered alloys ${ }^{10}$ and the divacancy mechanism in the fcc random alloy. ${ }^{11}$ On application to diffusion problems where the random alloy is a reasonable model to employ, the original sum-rule relation for the isolated vacancy mechanism results in very considerable and useful simplifications. The intent of the present paper is to review these applications. First, we summarize the random alloy model and the sum-rule itself. Next, we address intrinsic diffusion in multicomponent alloys, which is followed by a discussion of intrinsic diffusion in mixed alkali halides. Finally, we discuss

$\dagger$ Presented at the 85th Bunsen Colloquium on "Atomic Transport in Solids: Theory and Experiment", Gießen, Germany, October 31, 2003. demixing of multicomponent mixed oxides in oxygen potential gradients and electric fields.

\section{The random alloy model}

The sum-rules that have been found so far have been associated with various mechanisms in the random alloy model or variants/extensions of this model. It is appropriate therefore to review very briefly the random alloy model first. In this model, the $N$ atomic components are distributed randomly. The defects, e.g. vacancies, divacancies, dumb-bell interstitials are also distributed at random. Many diffusion kinetics formalisms are restricted to the case where the defects are at vanishingly small concentrations but this is not necessarily a restriction for the various sum-rules.

We will use focus on diffusion via the agency of isolated vacancies. The atom-vacancy frequencies, notated as $w_{i}$, can be conceptualized in one of two ways. On the one hand, one can consider them simply as explicit frequencies that depend only on the species of atom and not on the surroundings. For example, $w_{\mathrm{A}}$ simply then represents the basic vacancyatom frequency of a given $\mathrm{A}$ atom at all compositions. On the other hand, one can consider the $w_{i}$ as representing an average frequency at a given composition. ${ }^{5,12}$ In other words, $w_{\mathrm{A}}$ represents the average frequency of a given $\mathrm{A}$ atom as it migrates through the lattice sampling the various environments. Since the average environment of an atom will change with composition, then $w_{\mathrm{A}}$ can also be expected to change with composition.

The random alloy model with isolated vacancies can of course be used to describe concentrated substitutional alloys. $^{5,12,13}$ The same model can also be used to describe diffusion on the cation sublattice in mixed transition metal oxides or mixed alkali halides with intra-sublattice jumps. ${ }^{14,15} \mathrm{~A}$ straightforward extension of the model can also be used to describe diffusion in intermetallic compounds where each sublattice is a random alloy but at a different composition. ${ }^{10,16-18}$ In that case, both inter- and intra-sublattice jumps can be introduced.

\section{The sum-rule (isolated vacancy mechanism) ${ }^{4,7}$}

For the hopping model, the phenomenological coefficients are conveniently partitioned into a correlated part (a correlation 
function, frequently called the collective correlation factor) and an uncorrelated part:

$$
L_{i j}=f_{i j}^{(j)} L_{j j}^{(0)}
$$

where $f_{i j}^{(j)}$ is the collective correlation factor and $L_{i j}^{(0)}$ is the uncorrelated phenomenological coefficient. In general, both diagonal and off-diagonal phenomenological coefficients have non-trivial collective correlation factors. A result from linear response theory for the random alloy model is that the collective correlation factor can be written as:

$$
f_{i j}^{(j)}=\delta_{i j}-\frac{2 w_{i}}{c_{V} c_{j}} h_{V i: V j}
$$

where

$$
h_{i j: i_{0} j_{0}}=\sum_{s} \frac{s \cdot s_{0}}{s^{2}} \sum_{1} p_{\gamma}^{(i)}(1) p_{\gamma}^{(j)}(1-s) \Theta_{\gamma \beta}
$$

and

$$
\Theta_{\gamma \beta}=\Theta_{\gamma \beta}\left(1_{0}, 1_{0}-s_{0} ; i_{0}, j_{0}\right)=p_{\beta}^{\left(i_{0}\right)}\left(1_{0}\right) p_{\beta}^{\left(j_{0}\right)}\left(1_{0}-s_{0}\right) \int_{0}^{\infty} G_{\gamma \beta}(t) \mathrm{d} t
$$

where $\boldsymbol{s}$ and $\boldsymbol{s}_{0}$ are the nearest neighbour jump vectors and functions such as $p_{\gamma}^{(i)}$ (I) are the site occupancy variables $\left(p_{\gamma}^{(i)}\right.$ (l) equals 1 if site $\mathbf{l}$ is occupied in configuration $\gamma$ by one atom (or vacancy) of species $i$ and is zero otherwise) and $G_{\gamma \beta}(t)$ is the conditional probability that a system known to be in thermodynamic equilibrium and in the state $\beta$ will be in configuration $\gamma$ after time $t$. In eqn. (3) summation over $\beta$ and $\gamma$ configurations is implied. By the definition of the functions $p_{\gamma}^{(i)}$ (I) the following must be true:

$\sum_{i} p_{\gamma}^{(i)}(1)=1$,

where $i$ can be any atomic species or vacancy;

If we make a summation over all $j$ in eqn. (3):

$$
\sum_{j} h_{i j: i_{0} j_{0}}=\frac{\left(s_{1}+s_{2}+\ldots+s_{z}\right) \cdot s_{0}}{s^{2}} \sum_{l} p_{\gamma}^{(i)}(l) \Theta_{\gamma \beta} \equiv 0
$$

Again, in eqn. (5) the summation over $\beta$ and $\gamma$ configurations is implied.

In a somewhat easier way it can be shown that

$$
h_{i i: i_{0} j_{0}}=0 .
$$

Then, eqns. (5) and (6) together give:

$$
\sum_{j \neq i} h_{i j: i_{0} j_{0}}=0
$$

Applying eqn. (7) to the definition of $f_{i j}^{(j)}$ (eqn. (2)), we soon arrive at the following sum-rule relation for an $N$-component alloy with the vacancy mechanism operating: ${ }^{4,7}$

$$
\sum_{i=1}^{N} f_{i j}^{(j)} w_{j} / w_{i}=1, \quad j=1, \ldots, N
$$

Restated in terms of the phenomenological coefficients themselves we have:

$$
\sum_{i=1}^{N} L_{i j} w_{j} / w_{i}=A w_{j} c_{j}, \quad j=1, \ldots, N
$$

where $A$ is a constant. In effect, the 'sum-rule' relates the phenomenological coefficients to the vacancy-atom exchange frequencies and, in doing so, reduces the number of independent phenomenological coefficients.

\section{Some applications of the sum-rule}

In this section, we review several applications in diffusion problems of the above sum-rule. The first application is concerned with intrinsic diffusion in binary and ternary metallic alloys. The second application centres on intrinsic diffusion in mixed alkali halides, whilst the third application is concerned with demixing of ternary and quaternary transition metal oxides in oxygen potential gradients or electric fields. In each case, the sum-rule affords a very considerable simplification in the analysis.

\subsection{Intrinsic diffusivities in the multi-component alloy ${ }^{19}$}

We consider the $N$-component alloy. We write the flux equations in the lattice frame as (assuming sources and sinks are sufficiently numerous that $\nabla \mu_{\mathrm{V}} \approx 0.0$ and that there are no external forces):

$$
J_{i}=-\sum_{j=1}^{N} L_{i j} \nabla \mu_{j}, \quad i=1, \ldots, N
$$

Using the Gibbs-Duhem relation these equations can be transformed into:

$$
J_{i}=-\sum_{j=1}^{N-1} n D_{i j}^{N} \nabla c_{j}, \quad i=1, \ldots, N
$$

where $D_{i j}^{N}$ are the intrinsic diffusivities. They are generally measured by way of inert marker shifts in the interdiffusion experiment. The usual method is to use the marker velocity in conjunction with the measurement of the interdiffusivity. ${ }^{3}$ In practice, measurements of the intrinsic diffusivities can be rather time consuming, especially in ternary alloys and, accordingly, the amount of intrinsic diffusion data is generally rather limited compared with the far more plentiful interdiffusivity data. For binary AB alloys, we simply state that this ratio can be expressed in terms of the phenomenological coefficients in the usual way; see, for example, Allnatt and Lidiard: ${ }^{4}$

$$
\frac{D_{\mathrm{A}}}{D_{\mathrm{B}}} \equiv \frac{c_{\mathrm{B}} L_{\mathrm{AA}}-c_{\mathrm{A}} L_{\mathrm{AB}}}{c_{\mathrm{A}} L_{\mathrm{BB}}-c_{\mathrm{B}} L_{\mathrm{AB}}}
$$

where $D_{\mathrm{A}}$ and $D_{\mathrm{B}}$ are the intrinsic diffusivities in the AB binary alloy. The sum-rule (eqn. (9)) implies immediately that:

$$
\frac{D_{\mathrm{A}}}{D_{\mathrm{B}}}=\frac{w_{\mathrm{A}}}{w_{\mathrm{B}}}
$$

Similarly, for ternary $\mathrm{ABC}$ alloys, we have from the sum-rule (eqn. (9)) that:

$$
\begin{aligned}
& \frac{D_{\mathrm{BA}}^{\mathrm{C}} D_{\mathrm{CB}}^{\mathrm{C}}-D_{\mathrm{BB}}^{\mathrm{C}} D_{\mathrm{CA}}^{\mathrm{C}}}{D_{\mathrm{AA}}^{\mathrm{C}} D_{\mathrm{BB}}^{\mathrm{C}}-D_{\mathrm{AB}}^{\mathrm{C}} D_{\mathrm{BA}}^{\mathrm{C}}}=\frac{w_{\mathrm{C}}}{w_{\mathrm{A}}} \\
& \frac{D_{\mathrm{AB}}^{\mathrm{C}} D_{\mathrm{CA}}^{\mathrm{C}}-D_{\mathrm{AA}}^{\mathrm{C}} D_{\mathrm{CB}}^{\mathrm{C}}}{D_{\mathrm{AA}}^{\mathrm{C}} D_{\mathrm{BB}}^{\mathrm{C}}-D_{\mathrm{AB}}^{\mathrm{C}} D_{\mathrm{BA}}^{\mathrm{C}}}=\frac{w_{\mathrm{C}}}{w_{\mathrm{B}}}
\end{aligned}
$$

where $D_{\mathrm{AA}}^{\mathrm{C}}$ etc. are the ternary intrinsic diffusivities in the $\mathrm{ABC}$ ternary alloy and $\mathrm{C}$ is, by convention, the dependent concentration variable. The formal absence of any correlation factors and the corresponding simplicity of these equations should be especially noted.

The general expression (for $N$ atomic components) is:

$$
\tilde{D}\left[\begin{array}{c}
D_{N 1}^{N} \\
D_{N 2}^{N} \\
\cdots \\
D_{N N-1}^{N}
\end{array}\right]=\left[\begin{array}{c}
w_{N} / w_{1} \\
w_{N} / w_{2} \\
\cdots \\
w_{N} / w_{N-1}
\end{array}\right]
$$

where $\boldsymbol{D}$ is a matrix with elements $D_{k l}^{N}$ and $k, l \neq N$.

As an example of application of this finding for an experimental binary alloy system, in Fig. 1a we show results for the measured ratio of the intrinsic diffusivities and therefore the ratio of the exchange frequencies (from eqn. (13)) for the $\mathrm{Ag} / \mathrm{Cd}$ system. ${ }^{20}$ With the ratio of the atom-vacancy exchange frequencies in hand it is possible to investigate what effect the 
a)

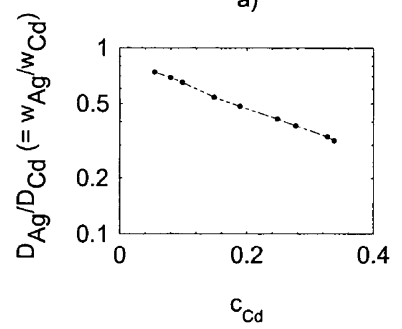

b)

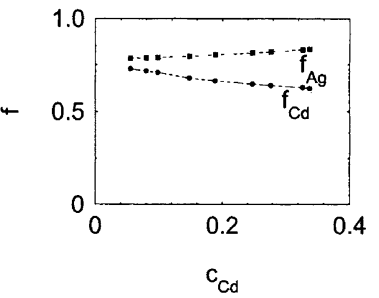

Fig. 1 (a) The ratio $D_{\mathrm{Ag}} / D_{\mathrm{Cd}}\left(=w_{\mathrm{Ag}} / w_{\mathrm{Cd}}\right)$ (plotted on a log scale) as a function of $c_{\mathrm{Cd}}$ at $873 \mathrm{~K} .{ }^{20}$ (b) Corresponding tracer correlation factors using the Moleko et al. formalism. ${ }^{22}$

interactions between the atoms might have on the exchange frequencies. As a very simple means of analysis, in the Ising alloy model (in a diffusion context) the interactions are nearly always introduced as the breaking of bonds as the atom jumps. This has the effect of augmenting or diminishing the original migration energy $U_{i}$. If we assume a purely random distribution of $\mathrm{A}$ and $\mathrm{B}$ atoms in the binary $\mathrm{AB}$ alloy the exchange frequencies can then be written in the spirit of the BraggWilliams approximation as:

$$
\begin{aligned}
& w_{\mathrm{A}}=\nu_{\mathrm{A}} \exp \left(-U_{\mathrm{A}} \beta\right) \exp \left(-\left(c_{\mathrm{A}} E_{\mathrm{AA}}+c_{\mathrm{B}} E_{\mathrm{AB}}\right)(z-1) \beta\right) \\
& w_{\mathrm{B}}=\nu_{\mathrm{B}} \exp \left(-U_{\mathrm{B}} \beta\right) \exp \left(-\left(c_{\mathrm{A}} E_{\mathrm{AB}}+c_{\mathrm{B}} E_{\mathrm{BB}}\right)(z-1) \beta\right)
\end{aligned}
$$

where $\nu_{\mathrm{A}(\mathrm{B})}$ are the attempt frequencies, $U_{\mathrm{A}(\mathrm{B})}$ are the hypothetical migration energies in the absence of interactions, $E_{\mathrm{AA}}$ is the interaction energy between a pair of nearest neighbouring A atoms, $E_{\mathrm{AB}}$ is the interaction energy between a pair of nearest neighbouring $\mathrm{A}$ and $\mathrm{B}$ atoms and $E_{\mathrm{BB}}$ is the interaction energy between a pair of nearest neighbouring B atoms, $z$ is the coordination, $c_{i}$ is the composition of component $i$ and $\beta=(k T)^{-1}$. If we make the further assumption that the attempt frequencies can be equated, then the ratio of the exchange frequencies $w_{\mathrm{A}} / w_{\mathrm{B}}$ is simply given by:

$$
\begin{aligned}
\frac{w_{\mathrm{A}}}{w_{\mathrm{B}}}= & \exp \left\{\left(-U_{\mathrm{A}}+U_{\mathrm{B}}-\left[c_{\mathrm{A}}\left(E_{\mathrm{AA}}-E_{\mathrm{AB}}\right)\right.\right.\right. \\
& \left.\left.\left.+c_{\mathrm{B}}\left(E_{\mathrm{AB}}-E_{\mathrm{BB}}\right)\right](z-1)\right) \beta\right\}
\end{aligned}
$$

For interaction energies that are independent of composition this implies therefore that $\ln \left(w_{\mathrm{A}} / w_{\mathrm{B}}\right)$ should be a linear function of composition at a given temperature. It can be seen that in the $\mathrm{Ag} / \mathrm{Cd}$ system the logarithm of this ratio is in fact close to being linear with composition, as suggested by eqn. (18). We hasten to add, however, that this is not always the case, particularly when a wide composition range is covered. Then it is likely that some dependence on composition of the interaction energies will be needed.

Of course, there is no reason why calculations on more sophisticated models using, for example, the embedded atom method or short time molecular dynamics cannot be used here. As with an exact kinetic treatment of the Ising model, the main obstacle would be to ensure that, given the large number of possible configurations, the frequencies are correctly ensemble-averaged. Unfortunately, at present such averaging procedures can only be performed satisfactorily with computer simulation such as kinetic Monte Carlo.

There has been a long tradition in diffusion research to determine tracer correlation factors, if possible, because these factors give rather direct information on the degree of correlation in the random walks of the atoms. Tracer correlation factors can of course be inferred from isotope effect experiments or from ratios of tracer diffusivities using a diffusion kinetics theory. ${ }^{5}$ In the present case, however, the tracer correlation factors can also be obtained by way of the ratio of the intrinsic diffusivities. With the ratio of the exchange frequencies in hand (from the ratio of the intrinsic diffusivities) the corresponding tracer correlation factors can be calculated readily using the random alloy diffusion kinetics formalisms of Manning 5 Holdsworth and Elliott ${ }^{21}$ or Moleko et al. ${ }^{22}$ Using the results of Fig. 1a and the latter formalism, which is the most accurate of the three, ${ }^{23}$ we present in Fig. 1b the corresponding values for $f_{\mathrm{Ag}}$ and $f_{\mathrm{Cd}}$. It can be seen that $\mathrm{Ag}$ atoms have the higher tracer correlation factors (they have the higher atom-vacancy exchange frequency) and are therefore less correlated in their motion that the $\mathrm{Cd}$ atoms.

\subsection{Intrinsic diffusivities in mixed alkali halides}

Now, let us consider the mixed alkali halide system $(\mathrm{A}, \mathrm{B}) \mathrm{Y}$, see for example Lindstrom ${ }^{15}$ and Allnatt and Lidiard. ${ }^{4}$ We assume that the cations $\mathrm{A}$ and $\mathrm{B}$ diffuse by isolated vacancies on the cation sublattice whilst the anions $\mathrm{Y}$ diffuse by isolated vacancies on the anion sublattice. The flux equations are (we assume that $q_{\mathrm{A}}=q_{\mathrm{B}}=-q_{\mathrm{Y}}$ ):

$$
\begin{aligned}
& J_{\mathrm{A}}=L_{\mathrm{AA}} X_{\mathrm{A}}+L_{\mathrm{AB}} X_{\mathrm{B}} ; \\
& J_{\mathrm{B}}=L_{\mathrm{AB}} X_{\mathrm{A}}+L_{\mathrm{BB}} X_{\mathrm{B}} ; \\
& J_{\mathrm{Y}}=L_{\mathrm{YY}} X_{\mathrm{Y}} .
\end{aligned}
$$

where for the internal forces we have:

$$
\begin{aligned}
& X_{\mathrm{A}}=-\nabla \mu_{\mathrm{A}}+e E_{i} ; \\
& X_{\mathrm{B}}=-\nabla \mu_{\mathrm{B}}+e E_{i} ; \\
& X_{\mathrm{Y}}=-e E_{i} .
\end{aligned}
$$

Upon application of the electro-neutrality conditions and the Gibbs-Duhem relation we find that: ${ }^{4,15}$

$$
J_{\mathrm{A}}=-D_{\mathrm{A}} N \nabla c_{\mathrm{A}}, J_{\mathrm{B}}=-D_{\mathrm{B}} N \nabla c_{\mathrm{B}}
$$

where

$$
\begin{aligned}
D_{A} & =\frac{\alpha k T c_{\mathrm{Y}}}{N c_{\mathrm{A}} c_{\mathrm{B}}}\left(\frac{\left.L_{\mathrm{AA}} L_{\mathrm{BB}}-L_{\mathrm{AB}}^{2}+L_{\mathrm{YY}}\left(L_{\mathrm{AA}} c_{\mathrm{B}}-L_{\mathrm{AB}} c_{\mathrm{A}}\right) / c_{\mathrm{Y}}\right)}{L_{\mathrm{AA}}+L_{\mathrm{BB}}+2 L_{\mathrm{AB}}+L_{\mathrm{YY}}}\right) \\
D_{B} & =\frac{\alpha k T c_{\mathrm{Y}}}{N c_{\mathrm{A}} c_{\mathrm{B}}}\left(\frac{\left.L_{\mathrm{AA}} L_{\mathrm{BB}}-L_{\mathrm{AB}}^{2}+L_{\mathrm{YY}}\left(L_{\mathrm{BB}} c_{\mathrm{A}}-L_{\mathrm{AB}} c_{\mathrm{B}}\right) / c_{\mathrm{Y}}\right)}{L_{\mathrm{AA}}+L_{\mathrm{BB}}+2 L_{\mathrm{AB}}+L_{\mathrm{YY}}}\right)
\end{aligned}
$$

and $N$ here is the number of lattice sites per unit volume. On application of the sum-rule (eqn. (9)) to the ratio of these intrinsic diffusion coefficients we soon find the interesting and simple result that:

$$
\frac{D_{\mathrm{A}}}{D_{\mathrm{B}}}=\frac{w_{\mathrm{A}}\left(w_{\mathrm{B}}+w_{\mathrm{Y}}\right)}{w_{\mathrm{B}}\left(w_{\mathrm{A}}+w_{\mathrm{Y}}\right)}
$$

where $w_{\mathrm{Y}}$ is the anion vacancy exchange frequency. For the limiting case $w_{\mathrm{Y}} \gg w_{\mathrm{A}}\left(w_{\mathrm{B}}\right)$, i.e. the anion mobility is very high, eqn. (23) then shows that the ratio of the intrinsic diffusion coefficients is simply given by $w_{\mathrm{A}} / w_{\mathrm{B}}$. Interestingly, this is the same result for the intrinsic diffusivities for the components $\mathrm{A}$ and $\mathrm{B}$ in the binary alloy described above (eqn. (13)). This comes about because the mobility on the anion sublattice can no longer determine the rate of interdiffusion. On the other hand, for the case where $w_{\mathrm{Y}} \ll w_{\mathrm{A}}\left(w_{\mathrm{B}}\right)$, i.e. the anion mobility is very low, according to eqn. (23) the ratio of the intrinsic diffusion coefficients is simply unity. This can be understood as follows. In the interdiffusion experiment with the diffusion couple AY-BY the essentially immobile anion sublattice requires the fluxes of the cations $\mathrm{A}$ and $\mathrm{B}$ to be equal and opposite. There is then no inert marker shift and the intrinsic diffusivities of the cations $\mathrm{A}$ and $\mathrm{B}$ are required to be equal. It also turns out that when the vacancy pair mechanism is employed, a different sum-rule is valid, but the same result as in eqn. (23) is still obtained. ${ }^{9}$ Put in another way, the result of measuring the ratio of the intrinsic diffusivities in the mixed alkali halide 
cannot differentiate between diffusion by isolated vacancies or vacancy pairs.

\subsection{Demixing of multicomponent oxides in an oxygen potential gradient or an electric field ${ }^{24-26}$}

As our final example of an application of the sum-rule, we consider steady state demixing in mixed transition metal oxides which are predominantly semi-conductors. For convenience, in the following we will analyse the quaternary mixed oxide $(\mathrm{A}, \mathrm{B}, \mathrm{C}) \mathrm{O}$ taking the rock-salt structure and consider demixing in an oxygen potential gradient and the electric field together. In these transition metal oxides the oxygen ions are essentially immobile at the temperatures of interest and so they largely act as 'spectators' for the cation diffusion processes. One end of the sample is exposed to a higher oxygen partial pressure (which, at a given temperature, determines the degree of nonstoichiometry and therefore the cation vacancy composition at this end) whilst the opposite end is exposed to a lower oxygen partial pressure, which again determines the (lower) cation vacancy composition. At high temperatures, where the cations are mobile, the vacancy gradient then gives rise to a vacancy flux across the sample and a cation flux in the opposite direction. An alternative way of obtaining a vacancy flux is to apply an electric field to the sample: under high current conditions there is a cation flux in direct response to the field and there is a vacancy flux in the opposite direction. At one end of the sample, in the direction of the cation flux, new oxide forms at the surface with oxygen coming from the gas phase. At the other end, excess oxygen ions evaporate and the oxide is lost. This results eventually in the sample moving with a steady state velocity $v$ in the direction of the higher oxygen partial pressure (or down-field if an electric field is imposed).

Electronic mobility is assumed far greater than the atomic mobilities and therefore is not rate determining in the diffusion of the cations. The cations A, B and C are assumed to be randomly mixed, i.e. the random alloy model. The flux equations are written as:

$$
J_{i}=-\sum_{j} L_{i j}\left(\nabla \mu_{j}-\nabla \mu_{V}-F_{j}\right), i, j=\mathrm{A}, \mathrm{B}, \mathrm{C}
$$

where $\mu_{i}$ is the chemical potential of component $i(\mathrm{~A}, \mathrm{~B}, \mathrm{C}$ or $\mathrm{V}$ (vacancies)), the $F_{j}$ are the electrical driving forces $(=q E$, where $q$ is the charge on the cation and $E$ is the electric field). For these mixed divalent ion oxides it is very reasonable to assume that $F_{\mathrm{A}}=F_{\mathrm{B}}=F_{\mathrm{C}}=F$.

The condition that leads to steady state of the demixing process can be expressed as: ${ }^{26}$

$$
J_{i}-v c_{i} N=0, i=\mathrm{A}, \mathrm{B}, \mathrm{C}
$$

where $c_{\mathrm{i}}$ is the composition of component $i$ (with respect to the cation sublattice so that $\left.c_{\mathrm{A}}+c_{\mathrm{B}}+c_{\mathrm{C}}+c_{\mathrm{V}}=1.0\right)$ and $N$ is the number of lattice sites per unit volume.

eqns. (24) and (25) then lead quickly to the result:

$$
\left[\begin{array}{l}
\nabla\left(\mu_{\mathrm{A}}-\mu_{\mathrm{V}}-F\right) \\
\nabla\left(\mu_{\mathrm{B}}-\mu_{\mathrm{V}}-F\right) \\
\nabla\left(\mu_{\mathrm{C}}-\mu_{\mathrm{V}}-F\right)
\end{array}\right]=\left[\begin{array}{l}
L_{\mathrm{AA}} L_{\mathrm{AB}} L_{\mathrm{AC}} \\
L_{\mathrm{AB}} L_{\mathrm{BB}} L_{\mathrm{BC}} \\
L_{\mathrm{AC}} L_{\mathrm{BC}} L_{\mathrm{CC}}
\end{array}\right]^{-1}\left[\begin{array}{l}
-c_{\mathrm{A}} \nu N \\
-c_{\mathrm{B}} \nu N \\
-c_{\mathrm{C}} \nu N
\end{array}\right]
$$

Eqn. (26) is the principal steady state demixing equation. It provides the basic relationships between the thermodynamic driving forces and the phenomenological coefficients as appropriate to the demixing experiment.

On substitution of the sum-rule (eqn. (9)) into eqn. (26) we soon obtain:

$$
\nabla\left(\mu_{i}-\mu_{\mathrm{V}}\right)=\frac{\nu c_{i} N}{L_{i i}^{(0)}}+F=-\frac{\nu N k T}{w_{i} c_{\mathrm{V}} K}+F, i=\mathrm{A}, \mathrm{B}, \mathrm{C}
$$

where we have written $L_{i i}^{(0)}=c_{v} c_{i} w_{i} K / k T$, where $K=N a^{2}$ and $a$ is the lattice parameter. Note the surprising absence of off-diagonal phenomenological coefficients in eqn. (27).

After converting the chemical potential gradients to composition gradients in the usual way: ${ }^{4}$

$$
\nabla\left(\mu_{i}-\mu_{\mathrm{V}}\right)=\sum_{j} \frac{\partial\left(\mu_{i}-\mu_{\mathrm{V}}\right)}{\partial c_{j}} \nabla c_{j}, \quad i, j=\mathrm{A}, \mathrm{B}, \mathrm{C}
$$

and assuming thermodynamic ideality, a condition which is, of course, appropriate for the random alloy model, then we can write that $\mu_{i}-\mu_{\mathrm{V}}=k T \ln \left(c_{i} / c_{\mathrm{V}}\right)$. This soon leads to the following coupled set of ordinary differential equations describing generally the steady-state composition profiles:

$$
\begin{aligned}
& \frac{\mathrm{d} c_{i}}{\mathrm{~d} \xi}=\frac{c_{i} \nu N}{c_{\mathrm{V}} K}\left(\sum_{j} \frac{c_{j}}{w_{j}}-\frac{1}{w_{i}}\right)+\frac{c_{i} c_{\mathrm{V}} F}{k T}, \quad i, j=\mathrm{A}, \mathrm{B}, \mathrm{C} \\
& \frac{\mathrm{d} c_{V}}{\mathrm{~d} \xi}=\frac{\nu N}{K} \sum_{i} \frac{c_{i}}{w_{i}}-\frac{c_{\mathrm{V}}\left(1-c_{\mathrm{V}}\right) F}{k T}, \quad i=\mathrm{A}, \mathrm{B}, \mathrm{C} .
\end{aligned}
$$

where $\xi$ represents a coordinate along the length of the moving sample. Eqn. (29) as derived here is exact for the randomly mixed system and for any value of the vacancy site fraction. These equations are appropriate for a 'closed' system where the cation vacancies within the material can respond to local kinetic conditions and forces.

On the other hand, if we impose the requirement that, for example, $c_{\mathrm{V}}=$ const (i.e. in the electric field case), then thermodynamic ideality conditions will give us $\nabla \mu_{\mathrm{V}}=0$. The final equation then is:

$$
\frac{\mathrm{d} \log \left(c_{i}\right)}{\mathrm{d} \xi}=-\frac{\nu N}{c_{\mathrm{V}} K w_{i}}+\frac{F}{k T}, \quad i=\mathrm{A}, \mathrm{B}
$$

with the relation $c_{\mathrm{C}}=1-c_{\mathrm{V}}-c_{\mathrm{A}}-c_{\mathrm{B}}$. This equation is appropriate for an 'open' system where the cation vacancies in the oxide are in communication with the external partial pressure(s) of oxygen. This would require extensive grain boundary diffusion and a small grain size.

In Fig. 2 we present results for the steady state demixed cation profiles of a ternary oxide $(\mathrm{A}, \mathrm{B}) \mathrm{O}$ in an oxygen potential gradient and for open and closed configurations. Monte Carlo results are shown for the closed system case only. Large differences are apparent in the demixed cation profiles for the cases of the open and closed systems. In real systems, careful fitting of the profiles with exchange frequency ratios obtained from tracer diffusivities (using, say, the kinetic theory of Moleko et $a l^{22}$ ) with supplementary information on the dependence of the cation vacancy concentration as a function

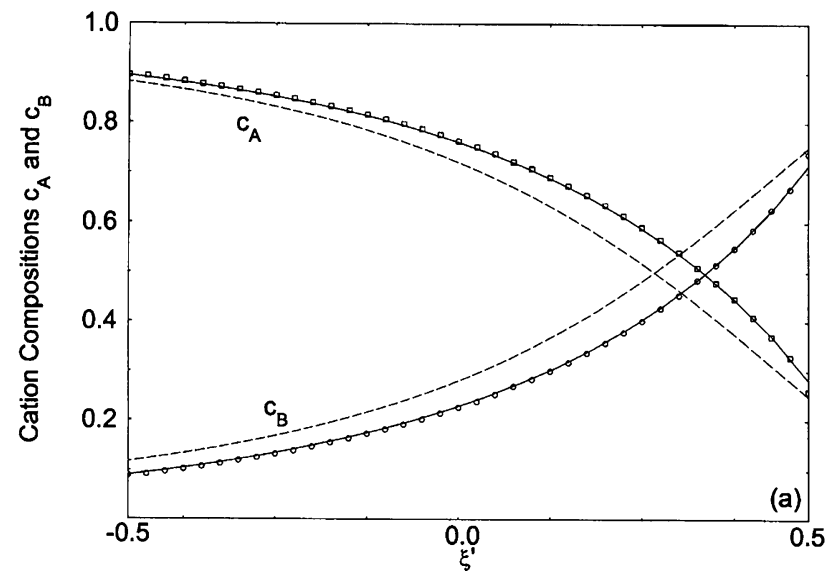

Fig. 2 The steady-state cation composition profiles for the case $w_{\mathrm{B}} / w_{\mathrm{A}}=0.1$. Symbols: Monte Carlo simulation results, solid lines: numerical solution for the closed system, dashed line: numerical solution for the open system (simple model). ${ }^{24}$ 


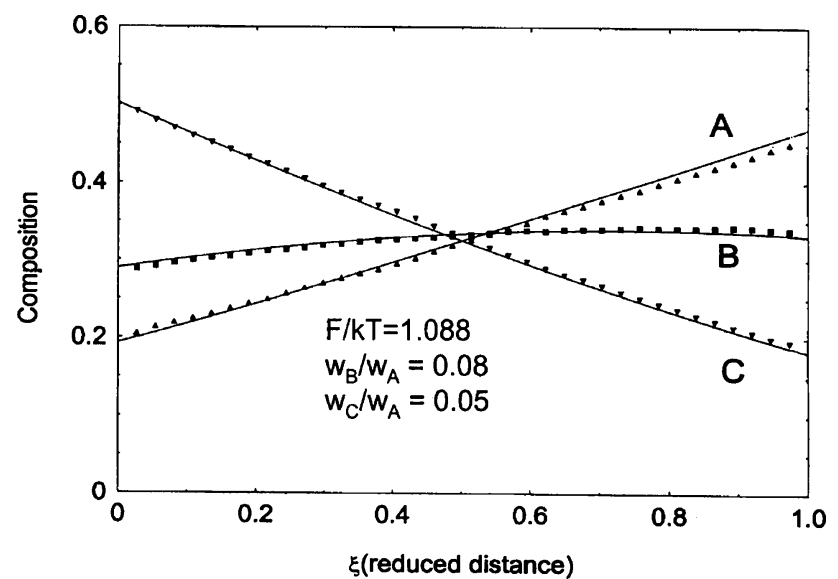

Fig. 3 The steady-state cation composition profiles in an electric field $F / k T=1.088$ for quaternary system $(\mathrm{A}, \mathrm{B}, \mathrm{C}) \mathrm{O}$ with $w_{\mathrm{B}} / w_{\mathrm{A}}=0.08$ and $w_{\mathrm{C}} / w_{\mathrm{A}}=0.05$. Symbols: Monte Carlo simulation results, solid lines: numerical solution for the closed system. Initial conditions: $c_{\mathrm{A}}=c_{\mathrm{B}}=c_{\mathrm{C}}=0.33$.

of (demixed) cation concentration, may suggest which possibility, i.e. open or closed is more appropriate to the experimental demixing situation. In Fig. 3, steady state demixing cation profiles are shown for a hypothetical $(\mathrm{A}, \mathrm{B}, \mathrm{C}) \mathrm{O}$ oxide after demixing in an electric field. Note the unusual shallow maximum in the concentration profile of the cation B (which has an atomvacancy exchange frequency intermediate between the other two).

\section{Conclusions}

In this paper, we have presented and discussed the sum-rule that relates the phenomenological coefficients in the multicomponent random alloy system with the isolated vacancy mechanism operating. We discussed application of this sumrule to intrinsic diffusion in multicomponent alloys, intrinsic diffusion in mixed alkali halides and demixing of multicomponent transition metal oxides in an oxygen potential gradient and an electric field. In each case, a very substantial simplification in the analysis was made possible on account of the sum-rule.

\section{Acknowledgements}

We wish to thank the Australian Research Council (Large Grants and Discovery Project Grants Schemes) for its support of this research. One of us (I.V.B.) wishes to thank the Australian Research Council for the award of Queen Elizabeth II and Professorial Fellowships.

\section{References}

1 R. E. Howard and A. B. Lidiard, Rep. Prog. Phys., 1964, 27, 161.

2 Y. Adda and J. Philibert, La Diffusion dans les Solides, Universitaires de France, Paris, 1966.

3 J. A. Philibert, Atom Movements: Diffusion and Mass Transport in Solids, Les Editions de Physique, Les Ulis, 1991.

4 A. R. Allnatt and A. B. Lidiard, Atomic Transport in Solids, Cambridge University Press, Cambridge, 1993.

5 J. R. Manning, Phys. Rev. B, 1971, 4, 1111.

6 A. B. Lidiard, Acta Metall., 1986, 34, 1487.

7 L. K. Moleko and A. R. Allnatt, Philos. Mag. A, 1988, 58, 677.

8 S. Sharma, D. K. Chaturvedi, I. V. Belova and G. E. Murch, Philos. Mag. Lett., 2000, 80, 65.

9 I. V. Belova and G. E. Murch, Philos. Mag., submitted.

10 I. V. Belova and G. E. Murch, Diffus. Defect Forum, 2001, 194-199, 547.

11 I. V. Belova and G. E. Murch, to be published.

12 J. R. Manning, Z. Naturforsch., 1971, 26a, 69.

13 H. Bakker, in Diffusion in Crystalline Solids, ed. G. E. Murch and A. S. Nowick, Academic Press, Orlando, FL, 1984, p. 189.

14 M. Martin, Ceram. Trans., 1991, 24, 91.

15 R. Lindstrom, J. Phys. C: Solid State Phys., 1974, 7, 3909.

16 I. V. Belova and G. E. Murch, Philos. Mag. A, 1996, 73, 117.

17 I. V. Belova and G. E. Murch, Philos. Mag. A, 1996, 73, 1223

18 I. V. Belova and G. E. Murch, Philos. Mag. A, 1996, 73, 1699.

19 I. V. Belova and G. E. Murch, Philos. Mag. Lett., 2001, 81, 661.

20 N. R. Iorio, M. A. Dayananda and R. E. Grace, Metall. Trans., 1973, 4, 1339.

21 P. C. W. Holdsworth and R. J. Elliott, Philos. Mag. A, 1986 54, 601 .

22 L. K. Moleko, A. R. Allnatt and E. L. Allnatt, Philos. Mag. A, 1989, 59, 141.

23 I. V. Belova, H. J. Braun and G. E. Murch, Acta Mater., 2003, 51, 1821 .

24 H. J. Braun, I. V. Belova and G. E. Murch, Philos. Mag. A, 2003, 83, 1855 .

25 H. J. Braun, I. V. Belova and G. E. Murch, Solid State Ionics, 2004, 167, 175.

26 H. Schmalzried, W. Laqua and P. L. Lin, Z. Naturforsch., 1974, 34a, 192. 